\title{
Using a rail simulation library to assess impacts of transit network planning on operational quality
}

\author{
E. M. Kanacilo ${ }^{1} \&$ N. van Oort ${ }^{2,3}$ \\ ${ }^{1}$ Faculty of Technology, Policy and Management, \\ Delft University of Technology, The Netherlands \\ ${ }^{2}$ Faculty of Civil Engineering and Geosciences, \\ Delft University of Technology, The Netherlands \\ ${ }^{3}$ HTM, Urban Public Transport Company, \\ The Hague, The Netherlands
}

\begin{abstract}
To achieve high operational quality in public transport, ex ante analysis should be made when planning the line network and timetable. This paper presents a Rail Simulation Library which is applicable for light rail and tram systems. This library is the core of a decision support environment to help designers assess the impact of network and timetable design on operational quality, e.g. speed and reliability. This model is used for designing a new light rail connection, RandstadRail, in The Hague. Different scenarios were run to calculate whether the line configuration and timetable were robust and stable. All scenarios consist of several stochastic processes like dwell time, driving time, turning time and waiting time at traffic lights. The model shows the results in both a tabular view as well as in an animation. The Rail Simulation Library proved to be suitable to forecast operations and to support the design of a line network and achievable timetable. By performing an ex ante analysis more robust and stable networks and timetables could be designed, thus facilitating a higher level for operational quality.
\end{abstract}

Keywords: simulation, reliability, network design, urban public transport, light rail, operations, forecasting. 


\section{Introduction}

When designing control systems for rail infrastructures (Harris and Godward [1] and Sussman [2]), control system designers aim at control strategies that will achieve a cost-effective and appropriate system to transfer passengers safely and reliably. Infrastructure capacity influences the quality of the transport service, not only in terms of reliability, with an infrastructure that provides enough vehicles to satisfy the demand, but also in terms of safety, where the transport of the required number of passengers can be done in a safe way.

Estimating infrastructure capacity can be very complex, as many factors influence the throughput of vehicles. Depending on the control strategies in use, the number of trams per hour that the infrastructure can support in a tram network can vary a lot. For example, giving priority to a tram line at crossings, or changing the speed limit along the rail network, will influence the travel time and consequently, it will influence the number of vehicles sharing the tracks during a certain time interval.

As advocated by Ceder [3], different aspects of the rail system should be analyzed at the same time, to exploit the system's capability to the greatest extent and maximize the system's productivity and efficiency.

Given the complexity of the task, simulation is an adequate method to support this what-if analysis during the control system design of a tram network and to help rail control designers in finding an appropriate set of control strategies that will increase the efficiency in using the infrastructure resources in relation to infrastructure capacity

Therefore, a library of simulation components proposed to enable schedule makers in assessing timetables at different system configurations before commissioning them.

In section 2 the design stages of public transport are described and the importance of reliability in public transport is explained. Section 3 presents the rail simulation library, which is used by HTM in a case study (section 4). Section 5 shows the conclusions and the paper ends with acknowledgements and references.

\section{Decision making in urban rail transport planning}

\subsection{Planning stages public transport}

In public transport design different levels could be distinguished: strategic, tactical and operational level. At the strategic level the network is designed. Expected rider ship, budget and geographical characteristics are input for the design of the service line network, existing of lines and main frequencies. At the strategic level the infrastructure network (curves, locations of switches, terminuses, etc.) is designed as well. This output, both types of networks, is used at the tactical level, at which the detailed timetable is constructed. Both the public timetable as the schedule for vehicles and crew is the output of this stage. 
After these two planning stages operations of the public transport services is executed. Enabling optimal design, feedback is necessary. Actual data is used to optimize both the network and timetable. Knowing the effect of design choices at these stages on performance at operational level is important. In case of new lines and infrastructure, no actual data is available, so feedback is impossible. Tools should be developed to help to create this feed forward loops.

\subsection{Reliability of public transport operations}

In Van Oort en Van Nes [4], a research of reliability of public transport in The Hague is presented. This research concludes that unreliability of the supplied service has a large effect on travel times of customers. Because of the relatively short journeys in urban areas the impact is quite large. In literature as well as in practice a lot of attention is paid to improving reliability at the operational level. Earlier research (e.g. Muller and Furth [5] and Wilson et al. [6]) suggests several solutions for this problem.

However, little attention has been paid so far to prevent these problems by taking reliability into account during the strategic and tactical planning phases of urban transit system design.

Figure 1 shows a visualization of reliability. Reliability is defined as the match between planning and operations. Operators offer a network and a timetable, which is their promise to the customer. Reliability defines to what extent they keep to this promise. Since two elements have to match, two ways of improving reliability are possible. By adjusting practice to planning in the one hand and fitting planning to practice on the other hand reliability will be increased. In both ways a higher level of service will be achieved.

During the planning stages prediction of the effect of the network design on reliability of operations should be possible. In this way optimal design could be made. The feasibility of the timetable should be investigated: which timetable is possible to execute and what are the effects of choices in both service network as infrastructure. This paper presents a tool with which the feasibility of the timetable and service network could be analyzed. Besides, the extra driving time due to interaction with other vehicles is calculated as well, enabling realistic timetables.

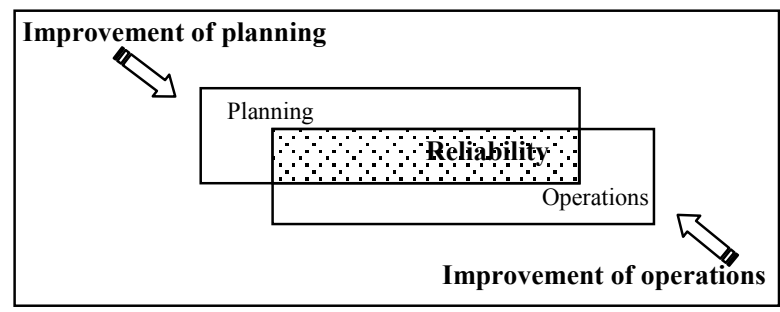

Figure 1: $\quad$ Service reliability in public transport and the role of planning and operations. 


\section{Rail simulation suite - framework}

In this paper is a library of simulation components organized in a service oriented way (Papazoglou [7]) proposed. In earlier research (Kanacilo and Verbraeck [8]), it is explained how the library is structured in service oriented architecture. Simulation components have been developed in Java and they are clustered by the type of services they offer (see figure 2).

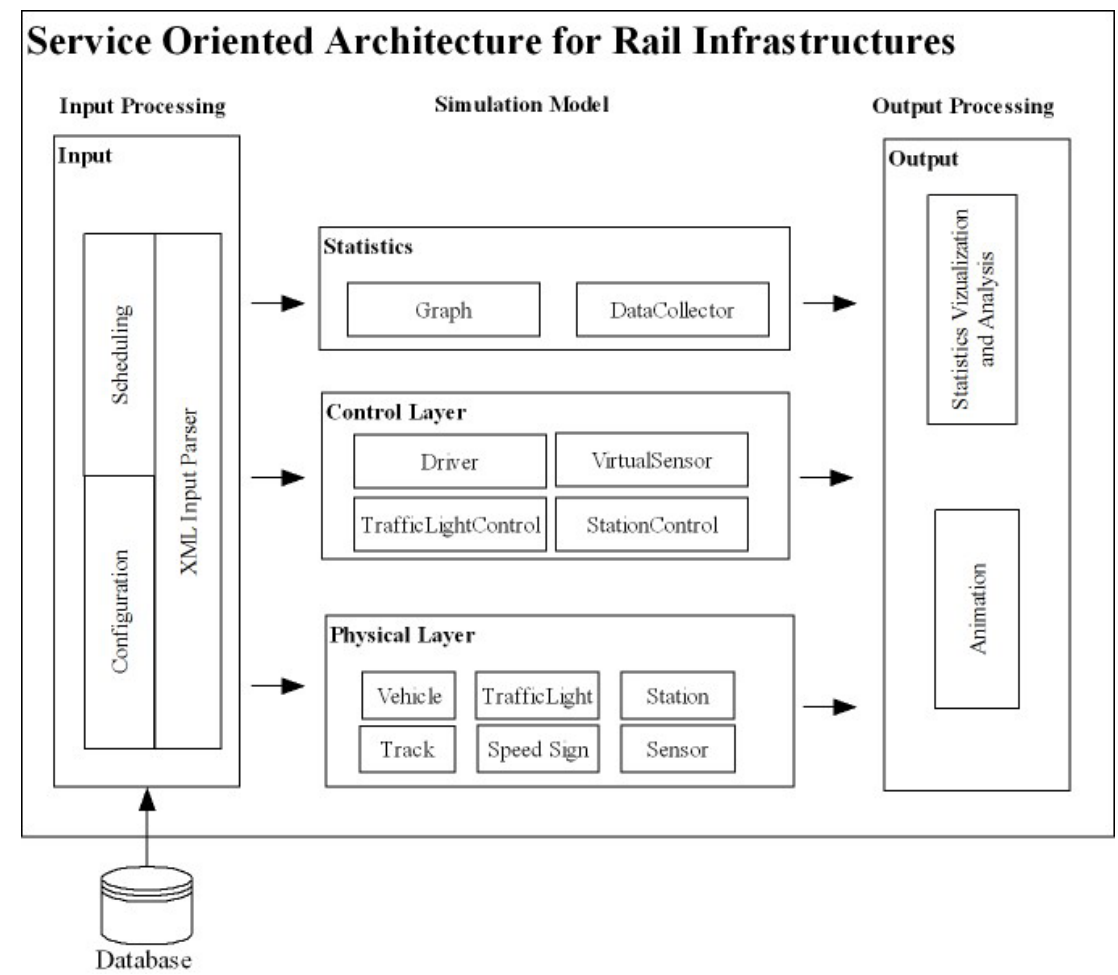

Figure 2: Rail simulation library.

The left side of the figure contains the components for input processing. These are components to check and validate input data, with the XML input file parser from which the whole model will be created as the most important component.

In the middle the components to create the simulation model are present. These are separated into layers:

- Physical Layer creates the physical objects, like for example traffic lights and sensors.

- Control Layer creates the logic behind each physical object, for example control part of the traffic light, which in fact defines the different types of traffic lights (different logic control). 
- Statistics Layer creates graphs and other objects to support the calculation of statistics during the simulation run.

On the right side there are the components to support output analysis, like the animation of object interaction and visualization of statistics. For more details about the architecture, see Kanacilo and Verbraeck [8].

The animation support users to have a better insight in the scenario being tested. Animation can be combined with GIS (Geographical Information Systems) drawings, like a city map to be used as background. These drawings help the user to quicker identify the analyzed section (based on the surrounding buildings and streets) and also helps in verifying whether scenario built is defined in the correct location. Figure 3 shows an example of the animation window.

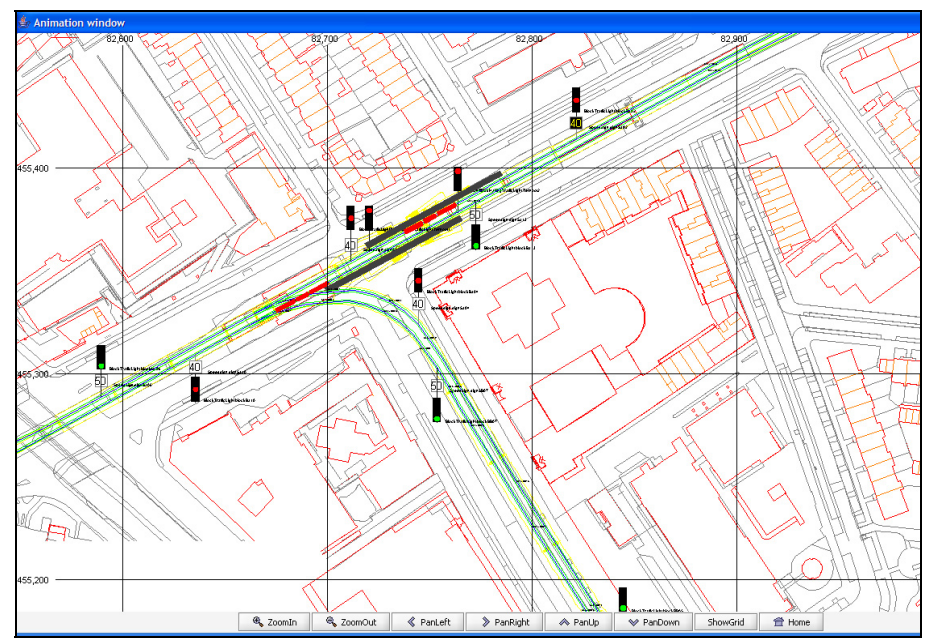

Figure 3: An example of the animation window.

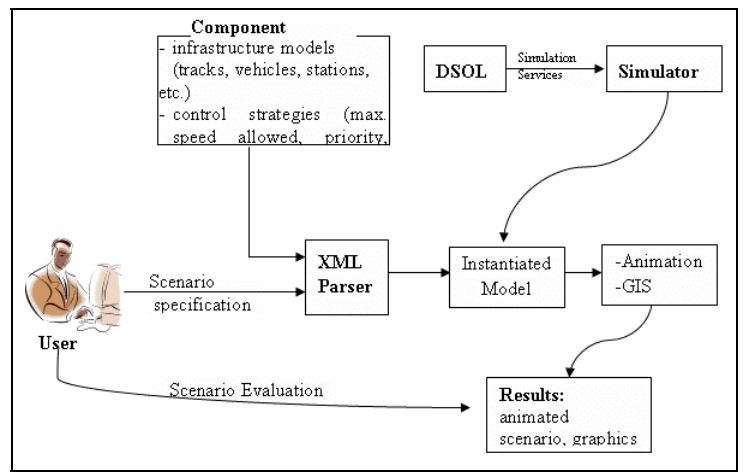

Figure 4: $\quad$ Use of simulation library. 
Figure 4 describes the relation among all these modules and how the testing environment works. First, the user specifies a scenario through an XML file. Before loading the model, the parser checks if all necessary data are entered and

if they are in the correct format to build the desired scenario. When the model has been created, it is ready to be simulated using the services provided by DSOL (Jacobs [9]). During the simulation run, graphics and statistics are gathered and are displayed to support the user in the process of deciding which strategy will be more appropriate for a certain system configuration.

\section{Case study "Monstersestraat"}

The tool presented in this paper is used in several projects of HTM, the urban public transport company in The Hague. RandstadRail is the new light rail connection between the cities of The Hague and Zoetermeer (Van Oort and Van Nes [10]). A former heavy railway lines is connected to two tramlines creating new direct connections into the heart of the city. RandstadRail offers high frequencies: 24 times an hour during rush hour. In the city, RandstadRail uses tracks, which are used by tram lines as well. Several junctions with tram lines exist as well. Because of the shared use of infrastructure and junctions in combination with high frequencies achieving a high level of service is a great challenge. The main bottlenecks were put into the model and the timetable was simulated to calculate the effect on driving time. Another goal was to determine whether achieving a high operational quality is possible. One of these bottlenecks is "Monstersestraat".

\subsection{Description network}

The rail configuration of the "Monstersestraat case" is illustrated in figure 5.

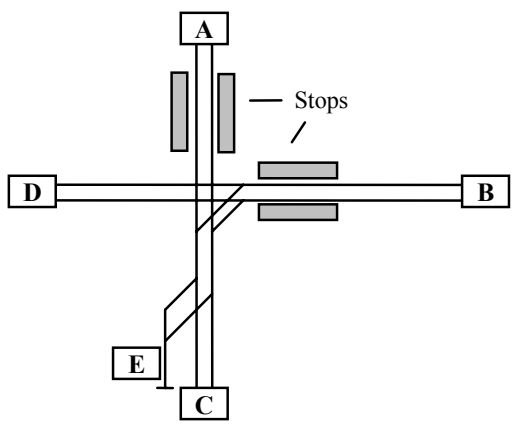

Figure 5: Rail configuration Monstersestraat.

At this location RandstadRail operates together with another tram line and crosses a tram line as well. Half of the RandstadRail vehicles terminate at this point on a tail track (at E). Table 1 gives the frequencies and routes of the 4 lines involved in this case. 
Table 1: $\quad$ Lines and frequencies.

\begin{tabular}{|c|c|c|c|}
\hline Line & Frequency $^{1}$ & From & $\mathrm{To}^{2}$ \\
\hline Tram 2 & 8 per hour & $\mathrm{B}$ & $\mathrm{D}$ \\
\hline Tram 11 & 6 per hour & $\mathrm{A}$ & $\mathrm{C}$ \\
\hline RandstadRail 4 & 6 per hour & $\mathrm{B}$ & $\mathrm{D}$ \\
\hline RandstadRail $4 \mathrm{~S}$ & 6 per hour & $\mathrm{B}$ & $\mathrm{E}$ \\
\hline
\end{tabular}

Both the junction with the other tram lines as the turning process at "E" could lead to disturbances. Simulation is used to assess the effects of these disturbances in a quantitative way. If the infrastructure of the junction and terminus prove not to be suitable, measures should be taken: infrastructure should be adjusted or extended or the service network should be changed. One possibility is not terminating half of the RandstadRail vehicles here, but ending this line elsewhere. Improving the schedule by introducing coordination or adding extra driving time is another possibility.

\subsection{Results analysis Monstersestraat}

The first step in the analysis is calculating of driving times of all lines. In a first simulation the free flow of all lines is measured: the speed and driving times in case of no delays due to other lines. After these free flow times have been determined the simulation of all lines operating together is executed.

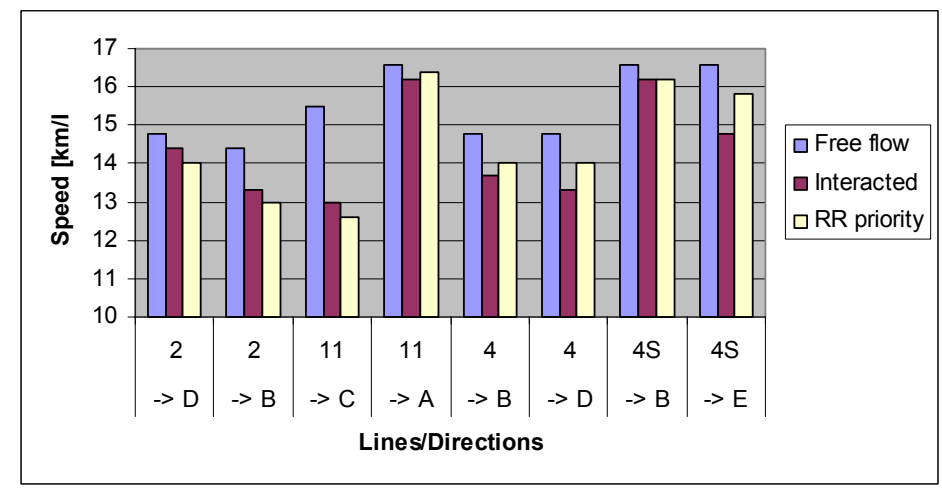

Figure 6: Operational speeds per line in three scenarios.

In this situation delays occur due to interference between lines: At the junction and terminus conflicting vehicles have to wait for each other. Figure 6 shows the operational speed (including dwelling and stopping) in this situation compared with the free flow situation. This figure shows that the decrease in speed is different per line and varies between about $2 \%$ and $16 \%$. 
The tool is also used to asses the impact of priority on driving times. If a conflict arises, priority is given to the RandstadRail lines 4 and 4S. Figure 6 also illustrates the effect on operational speeds. As to be expected, the velocity of RandstadRail increases, while line 2 suffers a decrease in speed. Surprisingly, line 11 improves in one direction because of the introduction of priority. Although, the effects of priority are low: the change in speed is about $2-7 \%$. The results of this simulation can be used to determine realistic driving times for the timetable. This enables the design of reliable timetables.

As stated in chapter 2, reliability of the operations is an important quality aspect of public transport. For RandstadRail a control philosophy is designed to achieve a high level of reliability (Van Oort and Van Nes [10]). An important aspect of this philosophy is to design infrastructure which enables high level of service. The simulation tool is used to asses the distribution of speeds to indicate the reliability of the service. Figure 7 shows the standard deviations of the speeds per line for three scenarios. It is shown that giving RandstadRail priority above the tramlines leads to a decrease in variability of the speeds and thus of driving times of these RandstadRail lines. But this improvement of reliability has a disadvantage too: the standard deviations of the tram lines increase. The results can be used to decide whether the infrastructure configuration or the service network restrict the operations too much.

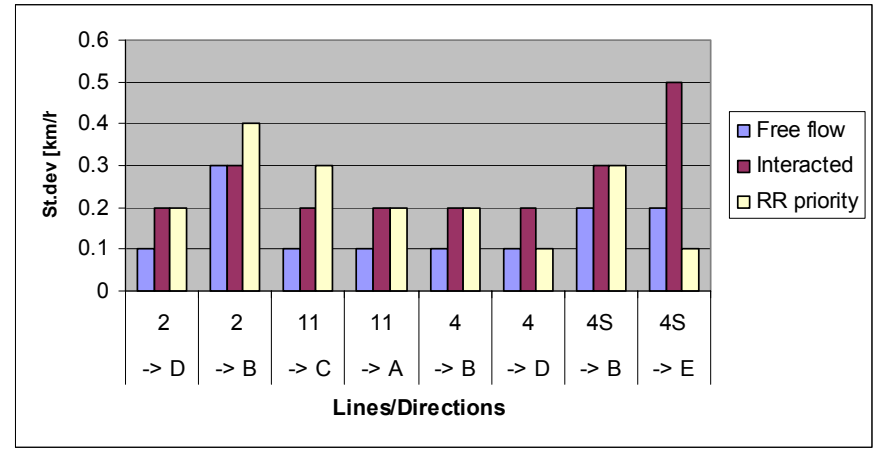

Figure 7: Operational speeds per line in three scenarios.

\section{Conclusions}

Reliability is a key quality indicator of urban public transport and the design of both network and timetable has a large impact on it. Choices made in the design process already determine which level of service can be maximally reached during operations. To assess the effect of network design on reliability a forecast tool is necessary. This paper describes a Rail Simulation Library. This tool enables prediction of the level of service of a certain urban rail configuration. A case study in The Hague shows the benefits of this tool: several scenarios are analyzed to calculate the effect of line and infrastructure networks on operational quality. This tool helps to optimize network design of urban public transport. 


\section{Acknowledgements}

This research is performed in cooperation with HTM, the public transport company in The Hague and Delft University of Technology, Faculty of Civil Engineering and Geosciences, department of Transport \& Planning and Faculty of Technology, Policy and Management, Systems Engineering Group. This research is supported by the Transport Research Centre Delft and Next Generation Infrastructure Research Program.

\section{References}

[1] Harris, N.G. and E.W. Godward, Planning Passenger Railways, Transport Publishing Company Ltd, Derbyshire, England, 1992.

[2] Sussman, J., Introduction to transportation systems, Norwood, MA., Artec House Inc. , 2000.

[3] Ceder, A., Advanced modeling for transit operations and service planning, Chapter Public Transport Timetabling and Vehicle Scheduling, Elsevier Science, pp. 31-57, 2003.

[4] Oort N. van and R. van Nes, Improving reliability in urban public transport in strategic and tactical design, 87th Annual Meeting of the Transportation Research Board, Washington DC, 2008.

[5] Muller Th.H.J. and P.G. Furth, Integrating bus service planning with analysis, operational control and performance monitoring, ITS 10th conference proceedings, Washington, D.C., 2000.

[6] Wilson, et al., Improving service on the MBTA green line through better operations control, Transportation Research Record 1361, TRB, National Research Council, Washington, D.C., pp. 296-304, 1992.

[7] Papazoglou, M. P., Service-oriented computing concepts, characteristics and directions, Proceedings of the 4th IEEE International Conference on Web Information Systems Engineering, IEEE Computer Society, pp. 3-12, 2003.

[8] Kanacilo, E. M. and A. Verbraeck, Simulation services to support the control design of rail infrastructures, Proceedings of the 2006 Winter Simulation Conference, ed. L. F. Perrone, F. P. Wieland, J. Liu, B. G. Lawson, D. M. Nicol, and R. M. Fujimoto, Piscataway, New Jersey: Institute of Electrical and Electronics Engineers, Inc., pp. 1372-1379, 2006

[9] Jacobs, P. H. M., The DSOL simulation suite - enabling multi-formalism simulation in a distributed context, Ph.D. thesis, Delft University of Technology, Delft, The Netherlands, 2005

[10] Oort, N. van and R. van Nes, RandstadRail: Increase in public transport quality by controlling operations, Proceedings Second International Seminar on Railway Operations Research, Hannover, 2007 